\title{
Investigation of field emission properties of carbon nanotube arrays defined using nanoimprint lithography
}

\author{
Sara M. C. Vieira, ${ }^{a)}$ Kenneth B. K. Teo, and William I. Milne \\ Department of Engineering, University of Cambridge, 9 JJ Thomson Avenue, Cambridge CB3 OFA, \\ United Kingdom \\ Oliver Gröning \\ EMPA Swiss Federal Laboratories for Materials Testing and Research, Feuerwerkerstrasse 39, \\ Thun CH-3602, Switzerland \\ Laurent Gangloff, Eric Minoux, and Pierre Legagneux \\ Thales Research and Technology, Route Départementale 128, 91767 Palaiseau Cedex, France
}

(Received 10 March 2006; accepted 18 May 2006; published online 12 July 2006)

\begin{abstract}
We demonstrate the use of nanoimprint lithography as an alternative low-cost fabrication route for the production of ordered arrays of individual carbon nanotube field emitters. A high emission site density of $4 \times 10^{5} \mathrm{~cm}^{-2}$ was observed and is well within the specification of the cathode for a field emission display. The measured field enhancement values from the geometry of the nanotubes were in reasonable agreement with the values obtained through electrical measurements. We also show that the distribution of the field enhancement factor is Gaussian, indicative of the presence of well ordered arrays of carbon nanotube field emitters. (C) 2006 American Institute of Physics.
\end{abstract}

[DOI: 10.1063/1.2219124]

We have previously shown that ordered arrays of individual multiwalled carbon nanotubes (MWCNTs) emitters could be achieved by combining lithography for growth catalyst definition and plasma enhanced chemical vapor deposition (PECVD) for MWCNT growth. ${ }^{1}$ In Ref. 1, electron beam lithography (EBL) was used to create patterns on the substrate followed by deposition of a conductive diffusion layer and the catalyst resulting, after growth, in localized spaced CNTs. The spacing between CNT emitters is necessary to avoid screening effects during field emission measurements. ${ }^{2}$ However, the EBL method previously used to create arrays of individual spaced MWCNTs is not suitable for mass production of emitters or microcathodes on a wafer/large scale due to the high cost of a high throughput EBL instrument. The need for low-cost high throughput selfassembly nanotechnology has resulted in the development of the nanoimprint lithography (NIL) technique. ${ }^{3,4}$ NIL can be used to produce any mark/space ratio (e.g., 1/100) in the pattern, in contrast to other lithographies (e.g., interferometry and nanosphere lithography) whose mark/space ratios are, to a certain extent, fixed. The resolution of NIL is not limited by effects of wave diffraction, scattering, and interference unlike photon or particle based lithographies. Additionally, NIL can pattern sub- $10-\mathrm{nm}$ features and has been used to produce patterns over $15 \mathrm{~cm} / 6 \mathrm{in}$. in diameter uniformly. In this letter, we demonstrate NIL as a route for producing large arrays of individual localized MWCNTs simultaneously for use in the production of self-assembly of CNT-based field emitters.

The NIL method is based on a hard master with predefined patterns pressed into a substrate with a soft layer of polymer resist such as polymethyl methacrylate (PMMA) at temperatures above the polymer glass transition $\left(T_{g}\right)$. The master is then released after a few minutes, resulting in pattern transfer followed by reactive ion etching (RIE) to remove the remaining residue in the patterned areas. Here, for

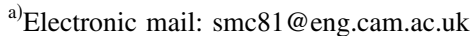

the substrate preparation, a $\mathrm{Si}(100)$ wafer was first immersed in hydrofluoric acid for approximately $1 \mathrm{~min}$. The acid removed the superficial layer of $\mathrm{SiO}_{2}$ as its presence causes electrical discharges during field emission-this is highly undesirable for field emission source devices. The wafer was then cleaned ultrasonically using acetone and isopropanol. Finally, the resist PMMA [950 K diluted in 4 vol \% anisole (A4)] was spun at $2300 \mathrm{rpm}$ and hard baked to remove any solvent still present $\left(140{ }^{\circ} \mathrm{C}\right.$ for $\left.1 \mathrm{~min}\right)$. The thickness of PMMA was measured to be $250-280 \mathrm{~nm}$ using a Dektak profilometer. The Si wafer employed for the master fabrication was cleaned in a similar way as the substrate. The resist (PMMA A4) was spun on the wafer, followed by e-beam exposure $(50 \mathrm{kV}, 50 \mathrm{pA})$, Mo sputtering, and then lift-off to yield a hard mask pattern of Mo round dots. Reactive ion etching using $\mathrm{CF}_{4}$ was then used to transfer the Mo pattern into the silicon as pillars, followed by removal of the Mo using wet acid etch. Note that EBL is only needed once in the overall process, that is, in the fabrication of the master. The master can then be used multiple times with cleaning to fabricate multiple wafers of emitters. Figure 1(a) shows a scanning electron microscopy (SEM) image (FEI Philips XL30, operating at $5 \mathrm{kV}$ ) of the master used for process optimization; the words NANO IMPRINT can be seen in reverse (mirror image). Figure 1(b) shows the master with $\mathrm{Si}$ pillars used for the CNT array fabrication with $10 \mu \mathrm{m}$ pitch, 270-280 nm diameter, and 230-250 nm height. The height of the Si pillars was less than the thickness of the resist polymer film, so that direct contact between master and substrate is avoided. During nanoimprinting, the PMMA can adhere to the master, making its subsequent removal difficult. To ensure effective release of the master, its surface was perfluorinated/made nonwetting by exposing the master to $1 H, 1 H-2 H, 2 H$ perfluorodecyltrichlorosilane (FDTS) in a dessicator for 2 days.

The actual nanoimprint experiments were performed with an Obducat ${ }^{\mathrm{TM}}$ nanoimprint tool. The pressure and temperature during imprint were varied from 40 up to 60 bars 


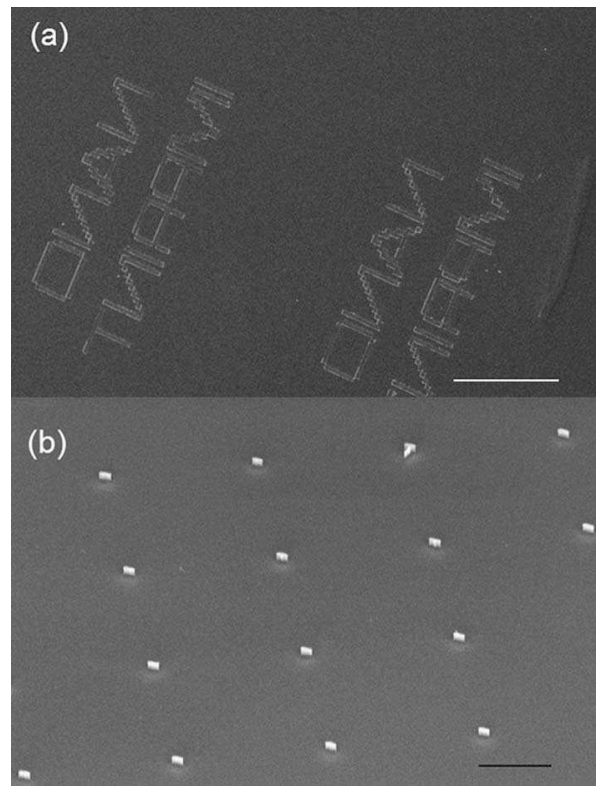

FIG. 1. (a) SEM image of the master used for NIL process optimization. The words NANO IMPRINT are seen inverted (mirror image). The scale bar is $20 \mu \mathrm{m}$. (b) SEM image of the master. The Si pillars have approximately $250 \mathrm{~nm}$ heights, approximately $270 \mathrm{~nm}$ diameters, and $10 \mu \mathrm{m}$ pitches. The tilt used was $45^{\circ}$ and the scale bar is $5 \mu \mathrm{m}$.

and from 160 up to $190{ }^{\circ} \mathrm{C}$, respectively. The release temperature was optimized at $70^{\circ} \mathrm{C}$ after $5 \mathrm{~min}$ in vacuum. The imprint was performed under vacuum to avoid the formation of air bubbles in the resist. The imprint temperature was increased approximately $80{ }^{\circ} \mathrm{C}$ above the glass transition temperature $\left(T_{g}\right)$ of PMMA to reduce the resist viscosity. PMMA residue still present after imprint was removed by RIE ( $\sim 0.2$ mbar), in 25:25 SCCM (standard cubic centimeter per minute) $\mathrm{O}_{2}$ :Ar with an etch rate of typically $3.5-4.0 \mathrm{~nm} \mathrm{~s}^{-1}$.

For CNT growth, we used an ITO diffusion barrier $(15 \mathrm{~nm})$ and a $\mathrm{Ni}(7 \mathrm{~nm})$ catalyst prepared by sputtering, which were then lifted off to form catalyst dot islands resembling the pattern on the master. The growth of aligned MWCNTs was performed by PECVD (Ref. 1) using acetylene and ammonia $\left(\mathrm{C}_{2} \mathrm{H}_{2}: 54 \mathrm{SCCM}, \mathrm{NH}_{3}: 200 \mathrm{SCCM}\right)$ at $725^{\circ} \mathrm{C}$. The bias for these set of experiments was increased slightly from the usual 600 to $630 \mathrm{~V}$ to produce CNTs with a smaller tip apex - this is due to the Ni catalyst being etched away by higher energy ions in the plasma as the CNT grows upwards.

For these needlelike structures, the field enhancement $(\beta)$ is related to the aspect ratio height/radius, in the form $\beta=h / r^{5}$. Figure 2(a) shows SEM images of CNTs grown using the master shown in Fig. 1(a); NANO IMPRINT letters formed by CNTs can be observed, where each letter consist of multiple aligned CNTs, made from a master bearing the pattern in mirror image. Figure 2(b) shows the SEM image of a $500 \times 500 \mu \mathrm{m}^{2}$ array of CNTs using the master shown in Fig. 1(b). Figure 2(c) shows an enlarged area of the array with CNTs spaced $10 \mu \mathrm{m}$ apart. The CNTs were 3-4 $\mu \mathrm{m}$ in height and $20-40 \mathrm{~nm}$ in tip radius. The inset in Fig. 2(c) shows an individual CNT with a Ni catalyst present. The measured tip radius was $30.5 \mathrm{~nm}$, and the tube was $3.53 \mu \mathrm{m}$ in height. Therefore, for this particular CNT the field enhancement factor $\left(\beta_{\mathrm{SEM}}=h / r\right)$ should be $\beta=3530 / 30.5$ $=115.7$. Downloaded 13 Jul 2006 to 129.169.10.56. Redistribution subject to AlP license or copyright, see http://apl.aip.org/apl/copyright.jsp

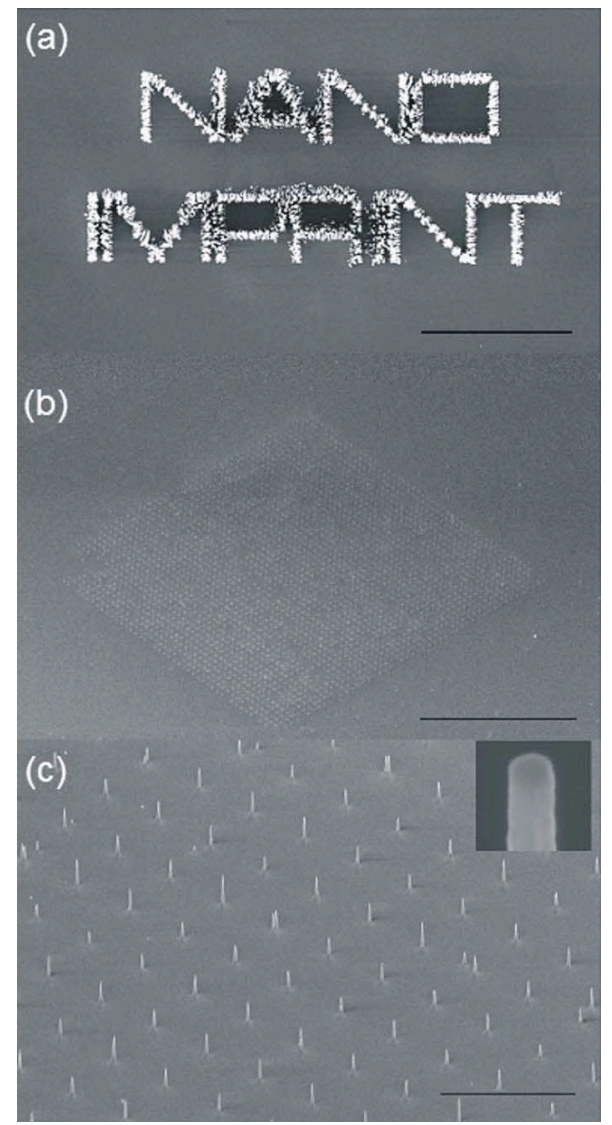

FIG. 2. SEM images of CNTs grown using NIL conditions: $185^{\circ} \mathrm{C}$, 50 bars, and $5 \mathrm{~min}$. Followed by etching $\mathrm{O}_{2}$ : $\mathrm{Ar}(25: 25 \mathrm{SCCM})$ and deposition of ITO $(15 \mathrm{~nm})$ and $\mathrm{Ni}(7 \mathrm{~nm})$. PECVD conditions: $725^{\circ} \mathrm{C},-630 \mathrm{~V}$, and $60 \mathrm{~min}$ run. (a) NANO IMPRINT words containing CNTs are seen. Scale bar is $20 \mu \mathrm{m}$. (b) Image showing an array of CNTs $(500$ $\times 500 \mu \mathrm{m}^{2}$ ). The SEM tilt was $45^{\circ}$ and the scale bar is $200 \mu \mathrm{m}$. (c) Closer image showing arrays of the NTs spaced by $10 \mu \mathrm{m}$. The tilt was $45^{\circ}$ and the scale bar is $20 \mu \mathrm{m}$. The small square indicates an individual MWCNT tip containing Ni catalyst with a measured $30.5 \mathrm{~nm}$ tip apex. The tilt used was $55^{\circ}$.

Scanning anode field emission microscopy (SAFEM) was performed to obtain information on the current-voltage $(I-V)$ characteristics, the field enhancement $(\beta)$, and the maximum emission current of single CNT emitters. In addition, statistical information on the distribution of field emission properties in the CNT field emitter array was measured using this technique, as described in Ref. 6. The sample was introduced in the SAFEM without prior treatment. Biasdistance curves were recorded at a constant emission current of $11 \mathrm{nA}$, with the tip centerd above an emitter. The measured $V$ - $d$ characteristic indicated a linear increase in voltage with increasing tip-sample distance with a corresponding

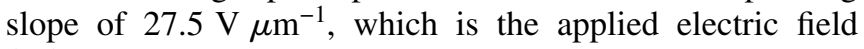
$\left(E_{a}\right)$. Furthermore, if we now fix $d$, and since $E_{a}=V / d$, for a fixed field of $27.5 \mathrm{~V} \mathrm{\mu m}^{-1}$ at $11 \mathrm{nA}$ obtained using $193 \mathrm{~V}$, one can determine the distance $d$ to be $7 \mu \mathrm{m}$.

The field at the tip of this emitter is related to the applied electric field by the relation $E_{\text {local }}=E_{a}^{*} \beta$. Assuming a work function of $\Phi=4.9 \mathrm{eV}$ previously determined for MWCNTs (Ref. 9) it can be predicted theoretically that an emission current of $11 \mathrm{nA}$ requires a local electric field of

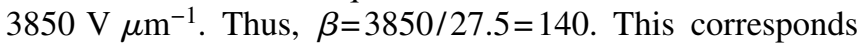
to a particular emitter with a $3.5 \mu \mathrm{m}$ height and a radius of curvature at the CNT apex of $25 \mathrm{~nm}$. From SEM measurements the average tube heights were $\sim 3.5 \mu \mathrm{m}$ and the aver- 

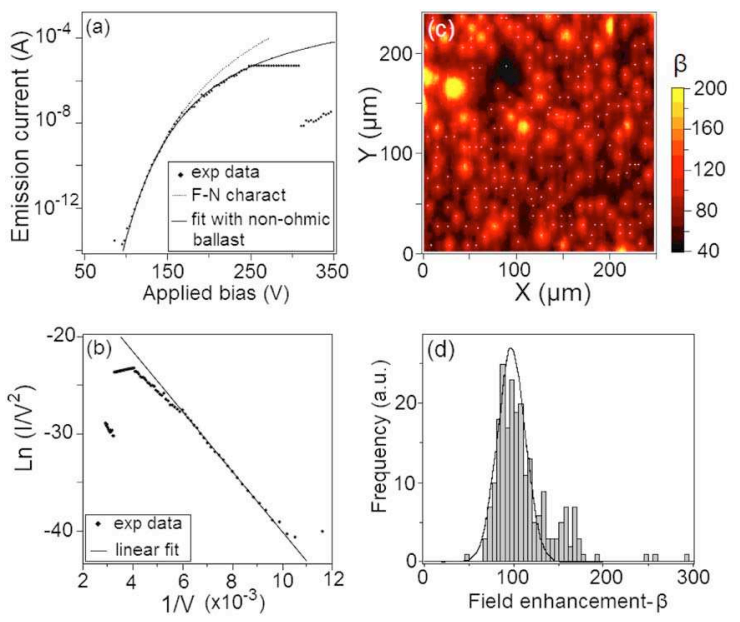

FIG. 3. (Color online) (a) The $I-V$ characteristics measured on a strong (high $\beta$ ) emitter. The distance of the tip over the single emitter was $7 \mu \mathrm{m}$. (b) Fowler-Nordheim plot of the $I-V$ characteristics with a linear fit to the low-current regime. (c) Field emission current mapping of the CNT array. Scan at a constant emission current of $11 \mathrm{nA}$ and a distance of $7 \mu \mathrm{m}$. Six scan lines with light discharges have been removed. The small white circles indicate the emitters found. (d) Field enhancement distribution function derived from the field enhancement map of (c). A Gaussian distribution is observed centerd at $\beta_{0}=97$ with FWHM of 37 .

age tip radius $\sim 30$. Therefore, the field enhancement value derived geometrically from the aspect ratio is in reasonable agreement with the Fowler-Nordheim (FN) (i.e., electrically measured) field enhancement.

Figure 3(a) shows the $I-V$ characteristics measured on an individual emitter by positioning the probe tip $7 \mu \mathrm{m}$ over a single CNT. The position of the single emitter has been determined from a scanning field emission map. From Fig. 3(a) one can observe that the experimental $I-V$ characteristics show typical behavior for the PECVD CNT emitters. In the low-current regime $(0.1 \mathrm{pA}-30 \mathrm{nA})$ the $I-V$ characteristics follow very nicely the Fowler-Nordheim curve like metallic emitter indicated by the dashed line in Fig. 3(a). However, for higher voltages and increased current deviation from the theoretical curve is observed, indicative of a current saturation of the emitter. This deviation can be explained by a voltage drop occurring both at the CNT/substrate contact and along the CNT length ${ }^{7,8}$ and is indicated by the black solid line. The flat line in the experimental data starting at $240 \mathrm{~V}$ is due to current limitation of the source measure unit which was set at $5 \mu \mathrm{A}$, and the abrupt decrease in current is due to the degradation of the emitter at high currents. Figure 3(b) shows the FN plot of the $I-V$ characteristic with a linear fit to the low-current regime.

To determine the frequency distribution of the field enhancement, the SAFEM is operated in constant current mapping mode. The bias $V(x, y)$ required to yield a constant emission current was measured as a function of the lateral tip position $(x, y)$ for a constant tip-cathode separation $(d)$. The local field at each emitter is thus $E_{\text {local }}=V(x, y)^{*} \beta(x, y) / d$. As determined previously, for a constant of $11 \mathrm{nA}, E_{\text {local }}$ should be $3850 \mathrm{~V} \mu \mathrm{m}^{-1}$ at a $7 \mu \mathrm{m}$ tip-sample distance $d$. Therefore, the field enhancement map $\beta(x, y)$ can be obtained from the relation $\beta(x, y)=3850^{*} 7 / V(x, y)$. Figure 3(c) shows the field enhancement map obtained using a tip-emitter distance of $7 \mu \mathrm{m}$. Each individual dot in the map represents a single emitter arranged on a $10 \mu \mathrm{m}$ pitch array, but, with different values of the field enhancement resulting in the intensity differences in the map. As can be seen from the map there are a number of holes where emitters are supposed to be active. The emitters on these sites could have been previously destroyed during prior measurements which determined the maximum current carrying capability of the emitters. In this map 225 emitters have been found. As we had fabricated emitters with a pitch of $10 \mu \mathrm{m}$, and the map size was $250 \times 240 \mu \mathrm{m}^{2}$, we expect approximately 600 emitters (i.e., $1 \times 10^{6} \mathrm{~cm}^{-2}$ ). Therefore, $37.5 \%$ of the nominal number of emitters could be imaged/were active corresponding to an emission site density (ESD) of $3.75 \times 10^{5} \mathrm{~cm}^{-2}$ for an ap-

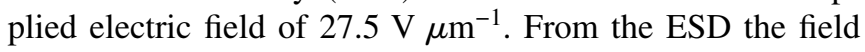
enhancement values for all the 225 emitters have been determined, and Fig. 3(d) shows the statistical distribution (histogram). The bulk of the emitters shows a Gaussian distribution centerd at $\beta_{0}=97$ with a full width at half maximum (FWHM) of 37 . Here the $\beta_{0}$ value obtained is consistent with the $\beta_{\text {SEM }}$ values for the CNTs derived from the aspect ratio measured in the SEM. The Gaussian distribution, indicated by the solid line in Fig. 3(d), strongly suggests the presence of regular emitter arrays with a certain deviation in emitter height and radius of curvatures. However, there are some emitters falling outside the Gaussian distribution. This asymmetry to higher field enhancement values is due to inhomogeneities related to the radius of curvature of the CNT emitter. Here, the maximum radius is given by the diameter of the CNT which varies in a rather narrow range. However, small protrusions on the spherical apex of the CNT can reduce the effective radius of the emitter significantly. As the field enhancement factor depends on the inverse of the radius, one can therefore observe factors considerably larger than what would be expected from the average radius of curvature of the emitter apex.

This work was funded by the EU project NANORAC. The authors thank Piers Andrew for perfluorinating the masters. One of the authors (S.M.C.V.) acknowledges support from the Portuguese Foundation of Science and Technology. Another author (K.B.K.T.) acknowledges the support of the Royal Academy of Engineering and Christ's College, Cambridge.

${ }^{1}$ K. B. K. Teo, S. B. Lee, M. Chhowalla, V. Semet, V. T. Binh, O. Gröning, M. Castignolles, A. Loiseau, G. Pirio, P. Legagneux, D. Pribat, D. G. Hasko, H. Ahmed, G. A. J. Amaratunga, and W. I. Milne, Nanotechnology 14, 204 (2003).

${ }^{2}$ L. Nilsson, O. Gröning, C. Emmenegger, O. Kuettel, E. Schaller, L. Schlapbach, H. Kind, J. M. Bonard, and K. Kern, Appl. Phys. Lett. 76, 2071 (2000).

${ }^{3}$ S. Y. Chou, P. R. Krauss, and P. J. Renstrom, Appl. Phys. Lett. 67, 3114 (1995).

${ }^{4}$ S. Y. Chou, P. R. Krauss, and P. J. Renstrom, Science 272, 85 (1996).

${ }^{5}$ F. Rohrbach, CERN Report 71, 5 (1971).

${ }^{6}$ L. Nilsson, O. Gröning, O. Kuettel, P. Gröning, and L. Schlapbach, J. Vac. Sci. Technol. B 20, 326 (2002).

${ }^{7}$ O. Gröning, O. M. Kuttel, Ch. Emmeneggar, P. Gröning, and L. Schlapbach, J. Vac. Sci. Technol. B 18, 665 (2000).

${ }^{8}$ L. Nilsson, O. Gröning, P. Gröning, O. Kuettel, and L. Schlapbach, J. Appl. Phys. 90, 768 (2001).

${ }^{9}$ E. Minoux, O. Gröning, K. B. K. Teo, S. H. Dalal, L. Gangloff, J. P. Schnell, L. Hudanski, I. Y. Y. Bu, P. Vincent, P. Legagneux, G. A. J. Amaratunga, and W. I. Milne, Nano Lett. 5, 2135 (2005). 